\title{
Light scattering by a prism and pyramid in the Rayleigh-Gans-Debye approximation
}

\author{
Konstantin A. Shapovalov \\ Department of Medical and Biological Physics, Krasnoyarsk State Medical Univ. named after Prof. V. F. Voyno-Yasenetsky, Partizana \\ Zheleznyaka Street, 1, Krasnoyarsk, 660022, Russia
}

\section{Email address:}

sh_const@mail.ru (K. A. Shapovalov)

\section{To cite this article:}

K. A. Shapovalov. Light Scattering by a Prism and Pyramid in the Rayleigh-Gans-Debye Approximation, Optics. Vol. 2, No. 2, 2013, pp. 32-37. doi: 10.11648/j.optics.20130202.11

\begin{abstract}
The general approach to finding the form factor of a compound particle and a system of particles in the Rayleigh-Gans-Debye (RGD) approximation is considered. The rotational-translational properties of light scattering amplitude in the RGD approximation are formulated. Using such properties, the analytical expressions for the amplitude of light scattering by a prism and pyramid with an arbitrary polygonal base in the RGD approximation are obtained. The phase functions of light scattering by a prism and pyramid in the RGD approximation are computed.
\end{abstract}

Keywords: Optically “Soft” Particles, Form Factor, Phase Function

\section{Introduction}

The light scattering and absorption of electromagnetic radiation is widely used in different branches of science and engineering for the study of structure and properties of inhomogeneous media. In recent years because of great significance such applications as optics of atmosphere and ocean, radio wave propagation, radio communication, physical chemistry of solutions and colloids, biophysics, laser biomedicine the theory and practice of light scattering techniques have been sufficiently developed [1-7]. Cloud droplets and atmosphere aerosols have a spherical shape in most cases, although particles of other shapes can exist due to various external influences $[3,5]$. For a spherical particle the well-known analytical solution or the Mie theory have been obtained by a separation of variables $[1,2]$. However, dust and soot particles, ice crystals of clouds have a strong nonspherical shape. For example, ice crystals of cirrus clouds are modeled by hexagonal prisms. In general, particles encountered in new practical applications are no longer considered spherical; they are nonspherical, nonrotational symmetric, inhomogeneous, coated, chiral or anisotropic. But if a particle has other than a regular geometrical shape, then it is difficult or impossible to solve the scattering problem analytically in its most general form that oblige to use numerical and approximate analytical methods.

Therefore, if particles of dispersion media are optically "soft" $|\mathrm{m}-1|<<1$, where $\mathrm{m}$ is a relative refractive index of light scattering particle (or particles are suspended in a medium with similar optical properties), then we can use suitable approximate methods of Rayleigh-Gans-Debye (RGD) or Anomalous Diffraction (AD) [1, 2, 4-9]. Note that the domain of validity of the RGD approximation is differed from the $\mathrm{AD}$ approximation $[2,4,7]$. The equations for the scattering and absorption properties of a column or prism with an arbitrary polygon base in the $\mathrm{AD}$ approximation have been obtained earlier $[10,11]$.

There have been some attempts to apply the RGD approximation to a particle of completely arbitrary shape and size, none of these has been truly satisfactory $[12,13]$, because of it leads us to a numerical solution by means of Fourier transformation. The analytical expressions are preferred by reason of they have more precise results and can serve as a basis for rigorous solution $[14,15]$.

Therefore, the purpose of this work is to give analytical expressions for the light scattering characteristics of a prism and pyramid with an arbitrary polygon base in the RGD approximation.

The paper consists of six sections. Section 2 contains a formulation of the light scattering problem, the main ideas of the method, and a brief description of the general approach for a composite or compound particle. Sections 3 and 4 contain some earlier results for a hexagonal cylinder and cone as particles similar to prism, pyramid, respectively, and a simple illustration of general approach for a system of 
several particles. Section 5 contains substantial and new results for a prism and pyramid in the RGD approximation. Section 6 contains concluding remarks.

\section{The Amplitude and Form Factor of a Compound Particle and System of Particles. General Approach}

Consider that a particle illuminates by a plane electromagnetic wave. Use integral expression of amplitude of light scattering in the RGD approximation (or the first Born approximation) in a scalar form $[7,16]$ :

$$
f(\theta, \beta)=\frac{k^{2}|\mathbf{P}|}{4 \pi} \int_{V}\left(m^{2}-1\right) \exp \left(i \mathbf{k}_{\mathbf{s}} \cdot \mathbf{r}\right) d V
$$

where $\mathbf{i}, \mathbf{S}$ are unit vectors along directions of the incident and scattering light respectively, $\mathbf{r}$ is the radius-vector of a point inside the particle, $\mathbf{k}_{\mathbf{s}}=k(\mathbf{i}-\mathbf{s}), \mathrm{k}=2 \pi / \lambda$ is the wave number, $\lambda$ is the wavelength of light, $\left|\mathbf{k}_{\mathbf{s}}\right|=2 k \sin (\theta / 2)$, $\theta$ is the angle between vectors $\mathbf{i}$ and $\mathbf{s}, \beta$ is the angle between axis $z$ and vector $\mathbf{k}_{\mathbf{s}},|\mathbf{P}|=\mid\left[-\mathbf{s} \times\left(\mathbf{s} \times \mathbf{e}_{\mathbf{i}}\right)\right], \mathbf{e}_{\mathbf{i}}$ is the unit vector along direction of the incident light polarization, (forth for brief text in a scalar form $|\mathbf{P}|=1$ ).

Note that the amplitude can be expressed another way in terms of the angles in spherical coordinates pointed direction of the incident $\theta \mathrm{i}, \phi \mathrm{i}$ and scattering light $\theta \mathrm{s}, \phi$ s separately:

$$
\begin{gathered}
k_{1}=k\left(\sin \theta_{i} \cos \phi_{i}-\sin \theta_{s} \cos \phi_{s}\right), \\
k_{2}=k\left(\sin \theta_{i} \sin \phi_{i}-\sin \theta_{s} \sin \phi_{s}\right), k_{3}=k\left(\cos \theta_{i}-\cos \theta_{s}\right), \\
k_{4}=\sqrt{k_{1}^{2}+k_{2}^{2}}, k_{s}=\sqrt{k_{1}^{2}+k_{2}^{2}+k_{3}^{2}} .
\end{gathered}
$$

The form factor in the RGD approximation $[1,2,4]$ for a homogeneous particle with the volume $V$ may be written as

$$
\Phi(\theta, \beta)=\frac{4 \pi f(\theta, \beta)}{k^{2}\left(m^{2}-1\right) V}=\frac{1}{V} \int_{V} \exp \left(i \mathbf{k}_{\mathrm{s}} \cdot \mathbf{r}\right) d V .
$$

Before we give some properties of light scattering amplitude note that the RGD approximation is valid when so-called "phase shift" of central ray $\Delta$ is much smaller compared with unity $(\Delta=2 \mathrm{ka}|\mathrm{m}-1|<<1$, where a is the longest dimension through the particle) $[1,2,4,7]$.

Firstly, for a composite particle, containing $q$ layers or distinct nonoverlapping regions $[1,2]$, we get

$$
\begin{gathered}
f(\theta, \beta)=\frac{k^{2}}{4 \pi}\left[\left(m_{q}^{2}-1\right) V_{q} \Phi_{q}(\theta, \beta)\right. \\
\left.+\sum_{j=1}^{q-1}\left(m_{j}^{2}-m_{j+1}^{2}\right) V_{j} \Phi_{j}(\theta, \beta)\right],
\end{gathered}
$$

where $\mathrm{j}$ is the number of layer (or region), $\mathrm{m}_{\mathrm{j}}$ is the relative refractive index of the $\mathrm{j}$ th layer, $\mathrm{V}_{\mathrm{j}}$ is its volume, $\Phi_{\mathrm{j}}(\theta, \beta)$ is the form factor of the $\mathrm{j}$ th layer.

Secondly, if a particle with the form factor $\Phi 0(\theta, \beta)$ shifts from center of coordinates to the position pointed by a vector $\mathbf{r}_{\mathbf{M}}$, then we can obtain the form factor as a multiplication by $\exp \left(i \mathbf{k}_{\mathbf{s}} \cdot \mathbf{r}_{\mathbf{M}}\right)$ :

$$
\begin{gathered}
\Phi_{M}(\theta, \beta)=\frac{1}{V} \int_{V} \exp \left(i \mathbf{k}_{\mathbf{s}} \cdot\left(\mathbf{r}+\mathbf{r}_{\mathbf{M}}\right)\right) d V \\
=\exp \left(i \mathbf{k}_{\mathbf{s}} \cdot \mathbf{r}_{\mathbf{M}}\right) \Phi_{0}(\theta, \beta) .
\end{gathered}
$$

Thirdly, if a particle rotates, for example, about axis OZ on Eulerian angle $\gamma$ and because Jacobian of transformation for such rotation in Eq. (2) is equal to 1, then we can obtain the form factor by transforming only expressions of $k_{1}, k_{2}$ into new position $k_{1}(\gamma), k_{2}(\gamma)$ as follows:

$$
\left(\begin{array}{l}
k_{1}(\gamma) \\
k_{2}(\gamma)
\end{array}\right)=\left(\begin{array}{cc}
\cos \gamma & -\sin \gamma \\
\sin \gamma & \cos \gamma
\end{array}\right)\left(\begin{array}{l}
k_{1} \\
k_{2}
\end{array}\right) .
$$

Thus, we establish rotational-translational properties of light scattering amplitude (not available in literature for the RGD approximation): shift or translation in Eq. (4), rotation (see Eq. (5)). Eqs. (3)-(5) provide us a convenient way to construct form factor in the RGD approximation for a compound particle and for a system of particles if the form factors of every particle are known.

\section{Circular and Hexagonal Cylinder}

The formulas for the calculation of light scattering characteristics: amplitude, phase function and others for a cylinder with a hexagonal section in the RGD approximation are obtained earlier by author $[17,18]$. For a homogeneous cylinder with height $\mathrm{H}$ and radius $\mathrm{R}$ the amplitude of light scattering $[1,4,17]$ is a well-known and may be written as

$$
f_{C Y L}=\frac{k^{2}\left(m^{2}-1\right) V_{C Y L}}{2 \pi} j_{0}\left(\frac{k_{3} H}{2}\right) \frac{J_{1}\left(k_{4} R\right)}{k_{4} R},
$$

where $V_{C Y L}=\pi R^{2} H$ is the volume of a circular cy-

linder, $\mathrm{J}_{1}(\mathrm{x})$ is a Bessel function of first order, $\mathrm{j}_{0}(\mathrm{x})=\sin \mathrm{x} / \mathrm{x}$ is a spherical Bessel function of zero order.

For a hexagonal cylinder (or a prism with hexagonal base) [17] the amplitude of light scattering is

$$
f_{H E X}=\frac{k^{2}\left(m^{2}-1\right) V_{H E X}}{6 \pi} j_{0}\left(\frac{k_{3} H}{2}\right)\left[F_{1}+F_{2}+F_{3}\right],
$$

where $V_{H E X}=\frac{3 \sqrt{3}}{2} R^{2} H$ is the volume of a hexagonal cylinder,

$$
\begin{gathered}
F_{1}=j_{0}\left(\frac{k_{1} R}{2}\right) j_{0}\left(\frac{k_{2} \sqrt{3} R}{2}\right) \\
F_{2}=\frac{1}{4}\left(1-\sqrt{3} \frac{k_{1}}{k_{2}}\right) j_{0}\left(\frac{\sqrt{3} R\left(k_{2}-\sqrt{3} k_{1}\right)}{4}\right) j_{0}\left(\frac{R\left(k_{1}+\sqrt{3} k_{2}\right)}{4}\right),
\end{gathered}
$$




$$
F_{3}=\frac{1}{4}\left(1+\sqrt{3} \frac{k_{1}}{k_{2}}\right) j_{0}\left(\frac{\sqrt{3} R\left(k_{2}+\sqrt{3} k_{1}\right)}{4}\right) j_{0}\left(\frac{R\left(k_{1}-\sqrt{3} k_{2}\right)}{4}\right) .
$$

For instance, let us assume that a system of three identical cylinders (not compulsory circular) paralleled $\mathrm{OZ}$ axis are illuminated along OY axis (see Fig. 1). Using Eqs. (4), (6), first cylinder of system located in the center of coordinates has the amplitude of light scattering $f_{\mathrm{CYL}}$, two others disposed on the same distance $d$ along OX axis to the left and to the right of first have the amplitude of light scattering $\exp \left(-i k_{1} d\right) f_{\mathrm{CYL}}$ and $\exp \left(i k_{1} d\right) f_{\mathrm{CYL}}$, respectively. Thus, using Eq. (3), the amplitude of a system of three identical cylinders is

$$
f_{3 C Y L}=\left(1+2 \cos \left(k_{1} d\right)\right) f_{C Y L} .
$$

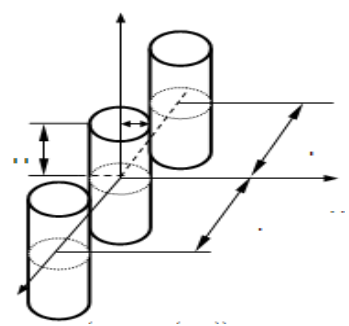

Figure 1. Geometry of light scattering by a system of three cylinders.

\section{Cone}

The amplitude of light scattering for a cone (Fig. 2) in case $k_{4}=0$, (in general case only expanded in series is available) [19] is

$$
\begin{aligned}
f_{\text {CONE }}= & \frac{k^{2} R^{2}\left(m^{2}-1\right)}{2 k_{3}}\left[h_{0}\left(k_{3} H\right)-j_{1}\left(k_{3} H\right)\right. \\
& \left.+i\left(1-h_{1}\left(k_{3} H\right)-j_{0}\left(k_{3} H\right)\right)\right],
\end{aligned}
$$

where $j_{1}(x)=\frac{\sin x-x \cos x}{x^{2}}, h_{0}(x)=\frac{1-\cos x}{x}$,

$h_{1}(x)=\frac{1}{2}+\frac{1-\cos x-x \sin x}{x^{2}}$ are spherical Bessel and

Struve functions of zero and first order, $\mathrm{k}_{3}=\mathrm{k}_{\mathrm{s}} \cos \beta$,

$\mathrm{k}_{4}=\mathrm{k}_{\mathrm{s}} \sin \beta$.

Furthermore, for case $k_{3} H=k_{4} R$ [19] the amplitude of light scattering by a cone in the RGD approximation is

$$
f_{\text {CONE }}=\frac{k^{2}\left(m^{2}-1\right) \exp \left(i k_{3} H\right) V_{C O N E}}{2 \pi}\left[f_{1}+i f_{2}\right]
$$

Where

$$
\begin{gathered}
f_{1}=\cos \left(k_{3} H\right) J_{1}\left(k_{3} H\right)+\sin \left(k_{3} H\right) J_{2}\left(k_{3} H\right), \\
f_{2}=\cos \left(k_{3} H\right) J_{2}\left(k_{3} H\right)-\sin \left(k_{3} H\right) J_{1}\left(k_{3} H\right), \\
V_{C O N E}=\pi R^{2} H / 3 .
\end{gathered}
$$

The amplitude of light scattering for a sphere in the RGD approximation $[1,2,4,6]$ is

$$
f_{S P H}=\frac{k^{2}\left(m^{2}-1\right) 3 V_{S P H}}{4 \pi} \frac{j_{1}\left(k_{s} R\right)}{k_{s} R}
$$

where $V_{S P H}=4 \pi R^{3} / 3$.

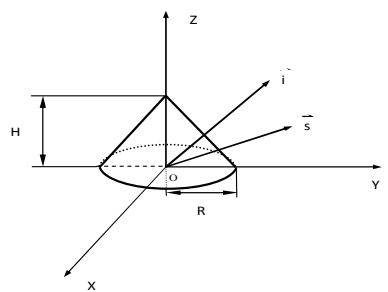

Figure 2. Geometry of light scattering by a cone.

The phase function [or element of scattering matrix $f_{11}$ ] for natural incident light (unpolarized or arbitrary polarized light) is calculated by formula

$$
f_{11}(\theta)=\left(\frac{1+\cos ^{2} \theta}{2}\right) k^{2}|f(\theta)|^{2}
$$

where $|f(\theta)|^{2}$ is a square of modulus of light scattering amplitude.

Further the phase function of light scattering is normalized on the value in a forward direction. And phase functions computed by Eqs. (6), (10), (11) for a cylinder, cone and sphere with relative refractive index $m=1.1+i 0.01$ are shown in Fig. 3.

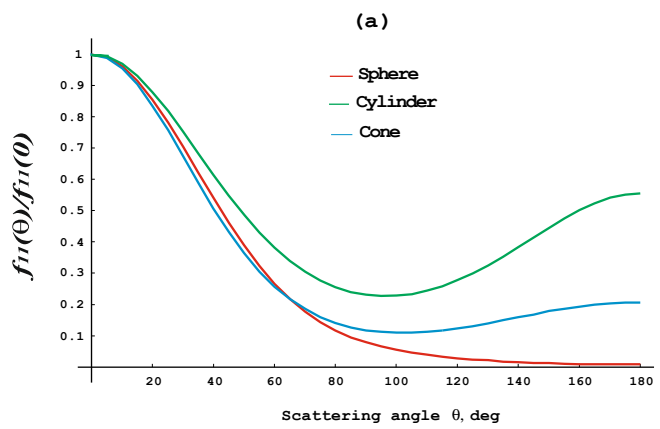

(b)

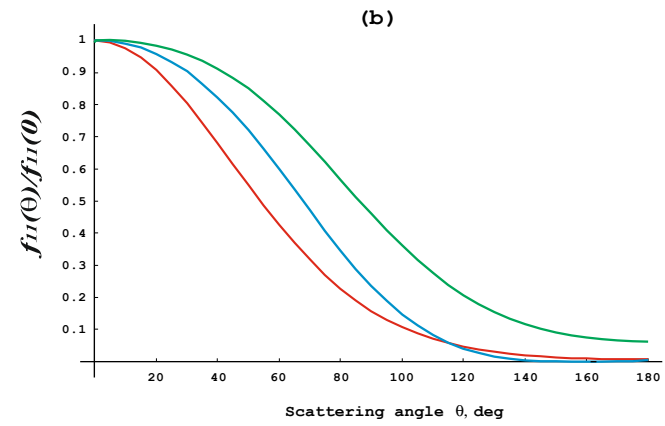

Figure 3. Normalized phase function $f_{11}(\theta) / f_{11}(0)$ vs. scattering angle $\theta$ for sphere, cylinder and cone in the RGD approximation provided $k R=2$, $k H=2$ and for the direction of incident light along the axis of symmetry (a) and perpendicular (b). 


\section{Prism and Pyramid with an Arbitrary Polygonal Base}

The analytical equations for the amplitude of light scattering in the RGD approximation by a prism (column) with an arbitrary polygonal base may be obtained using Eqs. (3), (5).

First of all, it's necessary to obtain expression of the amplitude of light scattering for an elementary polygon segment wedge (Fig. 4 (a)), having angle $\gamma$, radius of circumscribed circle $R$ and side $a$, with full height $H$.

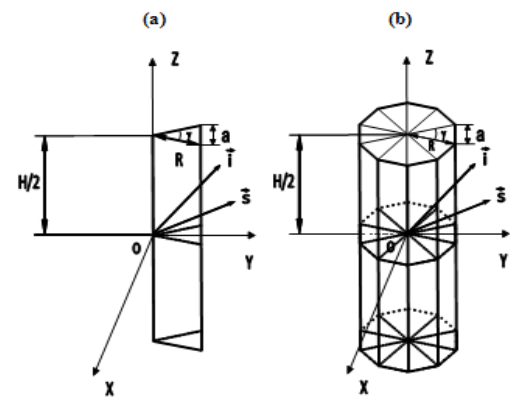

Figure 4. Geometry of light scattering by a wedge of polygonal prism (a) and whole polygonal prism (b), consisting of such wedges $(n=10)$.

Then, rotating wedges on angle $\gamma$-fold and summing amplitudes of wedges in new positions, using Eqs. (3),(5), we can get general amplitude of light scattering for a whole prism (see Fig. 4 (b)).

Thus, the amplitude for a polygonal prism segment wedge (see Fig. 4 (a)) is

$$
\begin{gathered}
f_{P M}=\frac{k^{2}\left(m^{2}-1\right) V_{P M}}{4 \pi k_{1} R \sin \gamma_{2}} j_{0}\left(k_{3} \frac{H}{2}\right) \\
\times\left[h_{0}\left(k_{5} R\right)-h_{0}\left(k_{6} R\right)+i\left(j_{0}\left(k_{6} R\right)-j_{0}\left(k_{5} R\right)\right)\right],
\end{gathered}
$$

where $V_{P M}=\frac{1}{2} H R^{2} \sin \gamma$ is the volume of a prism wedge, $\gamma=2 \pi / \mathrm{n}, \gamma^{2}=\gamma / 2, k_{5}=k_{2} \cos \gamma_{2}+k_{1} \sin \gamma_{2}$,

$k_{6}=k_{2} \cos \gamma_{2}-k_{1} \sin \gamma_{2}, \mathrm{R}$ is a radius of circumscribed circle, $\mathrm{n}$ is a number of segment of polygon, $\mathrm{j} 0(\mathrm{x}), \mathrm{h} 0(\mathrm{x})$ are spherical Bessel and Struve functions of zero order.

And the amplitude for a polygonal pyramid segment wedge (see Fig. 5 (a)) is

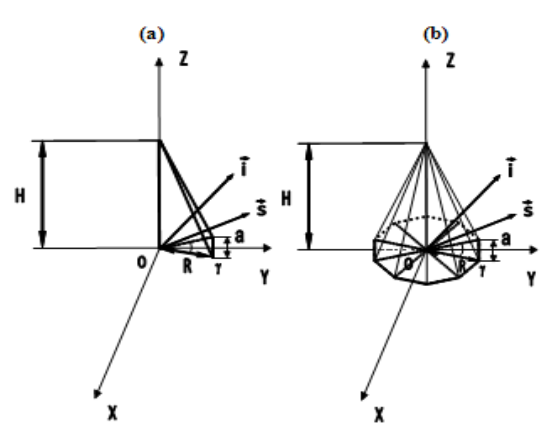

Figure 5. Geometry of light scattering by a wedge of polygonal pyramid (a) and whole polygonal pyramid (b).

$$
\begin{array}{r}
f_{P D}=\frac{k^{2}\left(m^{2}-1\right) 3 V_{P D}}{4 \pi k_{1} k_{3} R H \sin \gamma_{2}}\left[U\left(k_{6} R, 0\right)-U\left(k_{5} R, 0\right)\right. \\
\left.+U\left(k_{5} R, k_{3} H\right)-U\left(k_{6} R, k_{3} H\right)\right],
\end{array}
$$

where $V_{P D}=\frac{1}{6} H R^{2} \sin \gamma$ is the volume of pyramid wedge, $U(x, y)=\frac{\exp (i x)-\exp (i y)}{x-y}$.

Rotating about axis $\mathrm{OZ} n-1$ times the amplitude of light scattering by a wedge prism (11), using Eq. (5) and summing all terms, we obtain the amplitude for a whole prism as

$$
f_{\text {PRISM }}=\sum_{s=0}^{n-1} f_{P M}(s \gamma) .
$$

The special case $(n=6, \gamma=\pi / 3)$ for the amplitude of a hexagonal cylinder obtained from Eqs. (13),(15) is completely coincided with Eq. (7).

Analogically, rotating about axis OZ $n-1$ times the amplitude of light scattering by a wedge pyramid (14), using Eq (5) and summing all terms, we obtain the amplitude for a whole pyramid as

$$
f_{P Y R}=\sum_{s=0}^{n-1} f_{P D}(s \gamma)
$$

Note that if $n$ tends to infinity $(n \rightarrow \infty)$ then the amplitude in Eq. (15) converges to the amplitude of a circular cylinder Eq. (6), and the amplitude in Eq. (16) converges to the amplitude of a cone (see Eqs.(9), (10)). These main tendencies for Eqs. (15), (16) are successfully checked by a direct numerical comparison.

Thus, the amplitude of light scattering by a square base ( $n=4, \gamma=\pi / 2$ ) pyramid from (16) yields us

$$
f_{P Y R 4}=\frac{k^{2}\left(m^{2}-1\right) 3 V_{P D}}{2 \pi} \frac{\exp \left(i k_{3} H\right)}{k_{1} k_{2} R^{2}}\left[f_{3}+i f_{4}\right],
$$

Where

$$
\begin{gathered}
f_{3}=j_{0}\left(C_{1}\right)+j_{0}\left(C_{2}\right)-j_{0}\left(C_{3}\right)-j_{0}\left(C_{4}\right), \\
f_{4}=h_{0}\left(C_{1}\right)+h_{0}\left(C_{2}\right)-h_{0}\left(C_{3}\right)-h_{0}\left(C_{4}\right), R^{*}=R / \sqrt{2}, \\
C_{1}=k_{3} H+R^{*}\left(k_{2}-k_{1}\right), C_{2}=k_{3} H-R^{*}\left(k_{2}-k_{1}\right), \\
C_{3}=k_{3} H+R^{*}\left(k_{2}+k_{1}\right), C_{4}=k_{3} H-R^{*}\left(k_{2}+k_{1}\right) .
\end{gathered}
$$

And phase functions computed by formulas (15)-(17) for particles with relative refractive index $\mathrm{m}=1.1+\mathrm{i} 0.01$ are shown in Fig. 6. 
(a)

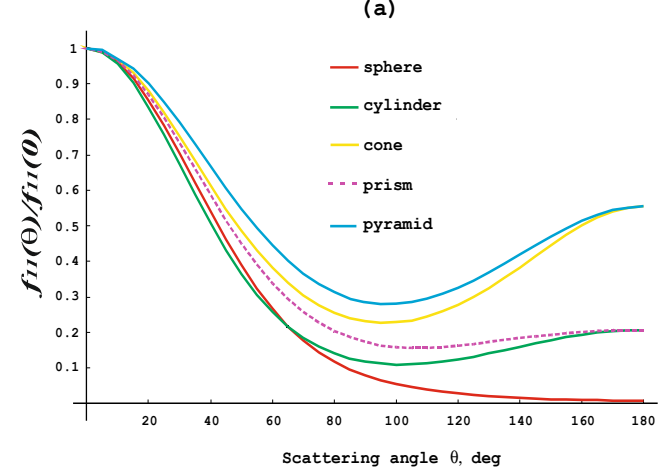

(b)

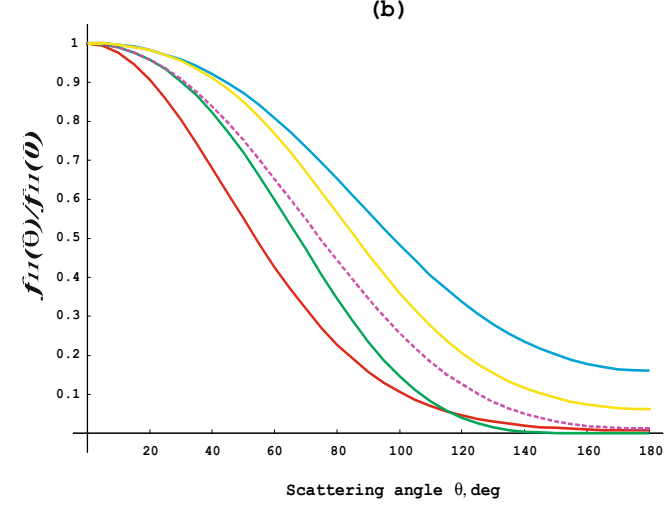

Figure 6. Normalized phase function $f_{11}(\theta) / f_{11}(0)$ vs. scattering angle $\theta$ for sphere, cylinder, cone, prism and pyramid provided $k R=2, k H=3, n=4$ and for the direction of the incident light along the axis of symmetry (a) and perpendicular (b).

\section{Conclusions}

Thus, we were discussed the general approach to obtaining of the form factor for a compound particle in the RGD approximation. The addition and rotational-translational properties of light scattering amplitude in the RGD approximation formulated herein allow us to construct the form factor for a system of several particles too. As a result of application of this technique formulas for the amplitude of light scattering by a prism and pyramid with an arbitrary polygonal base in the RGD approximation were obtained. The formulas obtained earlier for the amplitude of light scattering by a hexagonal cylinder and cone in the RGD approximation were presented too. In general, these expressions and technique may be also useful for the analytical evaluation of generalized parameters of polydisperse systems of particles, for the construction of new more precise approximations, for the comparisons with other solutions, techniques and for the further analysis of the validity's range of the RGD approximation.

\section{References}

[1] C.F. Bohren and D.R. Huffman, Absorption and Scattering of
Light by Small Particles. New York: John Wiley \& Sons, 1983.

[2] H.C. van de Hulst, Light Scattering by Small Particles. New York: John Wiley \& Sons, 1957.

[3] M.I. Mishchenko, J.W. Hovenier and L.D. Travis, Light Scattering by Nonspherical Particles: Theory, Measurements, and Applications. San Diego: Academic Press, 2000.

[4] M. Kerker, The Scattering of Light and Other Electromagnetic Radiation. New York, London: Academic Press, 1969.

[5] A.A. Kokhanovsky, Cloud Optics. Dordrecht: Springer, 2006.

[6] V.V. Tuchin, ed., Handbook of Optical Biomedical Diagnostics. vol. PM107. Bellingham: SPIE Press, 2002.

[7] V.N. Lopatin, A.V. Priezzev, A.D. Aponasenko, N.V. Shepelevich, V.V. Lopatin, P.V. Pozhilenkova and I.V. Prostakova, Methods of Light Scattering in Analysis of Dispersion Biological Media. Moscow: PhysMatLit, 2004. (in Russian).

[8] P. W. Barber and D.-S. Wang, "Rayleigh-Gans-Debye applicability to scattering by nonspherical particles," Appl. Opt. vol. 17, pp. 797-803, 1978.

[9] K.A. Shapovalov, "Light scattering by particles of toroidal shape in the Rayleigh-Gans-Debye approximation," Opt. Spectrosc., vol. 110, No.5, pp. 806-810, 2011.

[10] P. Chýlek and J.P. Klett, "Extinction cross section of non-spherical particles in the anomalous diffraction approximation," J. Opt. Soc. Am. A, vol. 8, pp. 274-281, 1991.

[11] P. Chýlek and J.P. Klett, "Absorption and scattering of electromagnetic radiation by prismatic columns: Anomalous diffraction approximation,” J. Opt. Soc. Am. A, vol. 8, pp. 1713-1720, 1991.

[12] V.M. Rysakov, "Light scattering by "soft" particles of arbitrary shape and size," J. Quant. Spectrosc. Radiat. Transfer, vol. 87, pp. 261-287, 2004.

[13] K. Muinonen, "Light scattering by Gaussian random particles: Rayleigh and Rayleigh-Gans approximations," J. Quant. Spectrosc. Radiat. Transfer, vol. 55, pp. 603-613, 1996.

[14] J.W. Shepherd and A.R. Holt, "The scattering of electromagnetic radiation from finite dielectric circular cylinders," J. Phys. A: Math. Gen., vol. 16, pp. 651-662, 1983.

[15] A.R. Holt, N.K. Uzunoglu and B.G. Evans, "An integral equation solution to the scattering of electromagnetic radiation by dielectric spheroids and ellipsoids," IEEE Trans. Antennas Propag., vol. 26, pp. 706-712, 1978.

[16] A. Ishimaru, Wave Propagation and Scattering in Random Media. New York: IEEE Press, 1997.

[17] K.A. Shapovalov, "Light scattering of cylindrical particles in Rayleigh-Gans-Debye approximation. 1. Rigorously oriented particles," Atmos. Oceanic. Opt., vol. 17, No. 4, pp. 350-353, 2004.

[18] K.A. Shapovalov, "Light scattering of cylindrical particles in Rayleigh-Gans-Debye approximation. 2. Randomly oriented particles," Atmos. Oceanic. Opt., vol. 17, No. 8, pp. 627-629, 2004. 
[19] K.A. Shapovalov, "Light scattering by particles with axis of symmetry in Rayleigh-Gans-Debye approximation," J. Sib. Fed. Univ. Math. Phys., vol. 5, No. 4, pp. 586-592, 2012. http://elib.sfu-kras.ru/bitstream/2311/3112/1/shapevalev.pdf (Open Access Journal in Russian). 